Supplement of Hydrol. Earth Syst. Sci., 25, 1785-1812, 2021

https://doi.org/10.5194/hess-25-1785-2021-supplement

(C) Author(s) 2021. CC BY 4.0 License.

(c) (i)

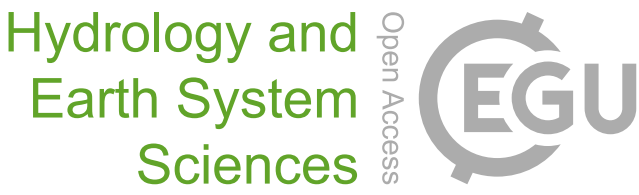

Supplement of

\title{
Exploring the regolith with electrical resistivity tomography in large-scale surveys: electrode spacing-related issues and possibility
}

\section{Laurent Gourdol et al.}

Correspondence to: Laurent Gourdol (laurent.gourdol@ list.lu)

The copyright of individual parts of the supplement might differ from the article licence. 


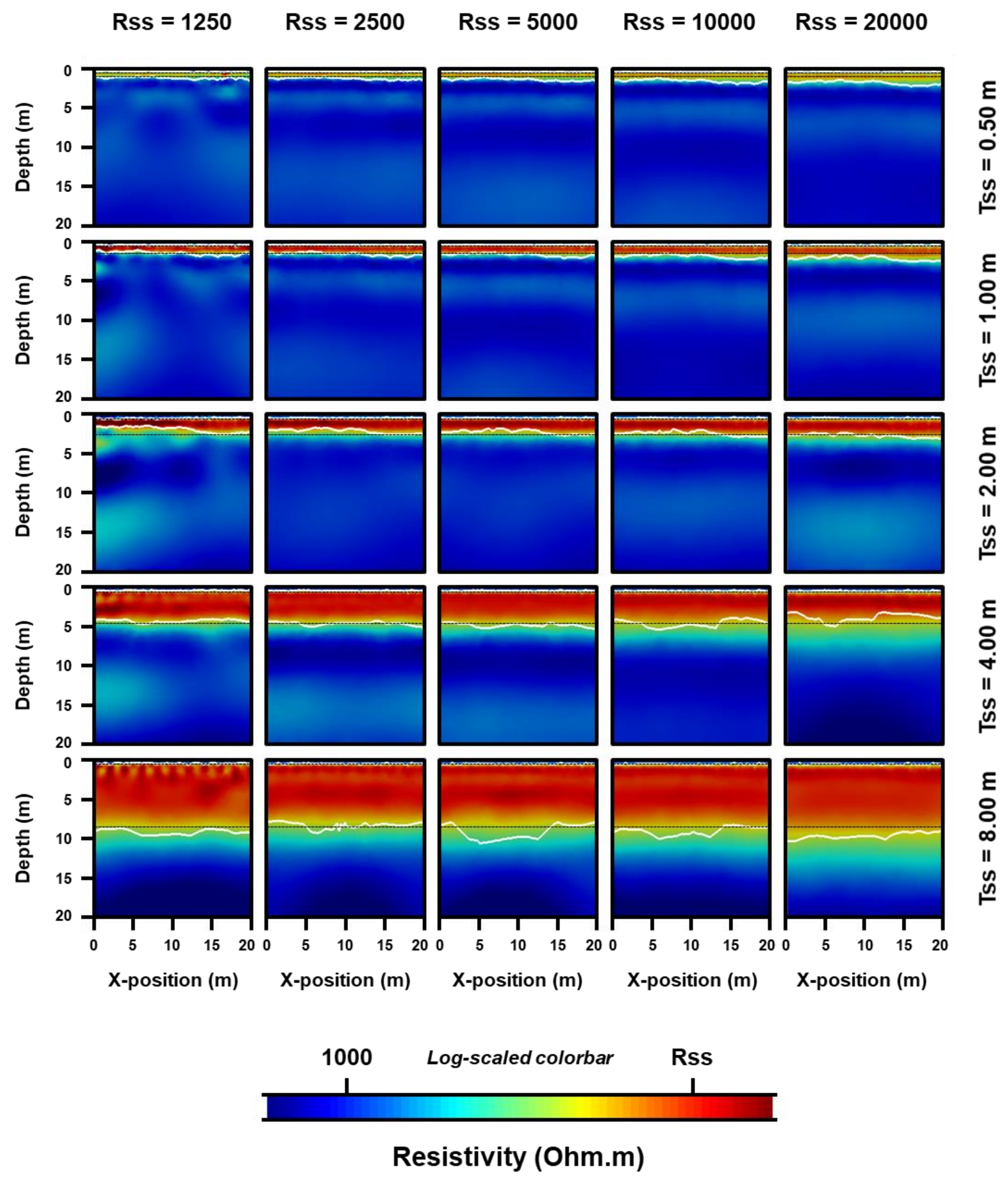

Figure S1: Results of inversion of the synthetic resistivity models (Rss and Tss values stand for the subsolum resistivity and thickness in the model, respectively; ERT-derived and true solum thickness and depth to bedrock interfaces are shown by white and black dashed lines, respectively) using the Wenner-Schlumberger array with an ES of $0.25 \mathrm{~m}$. 


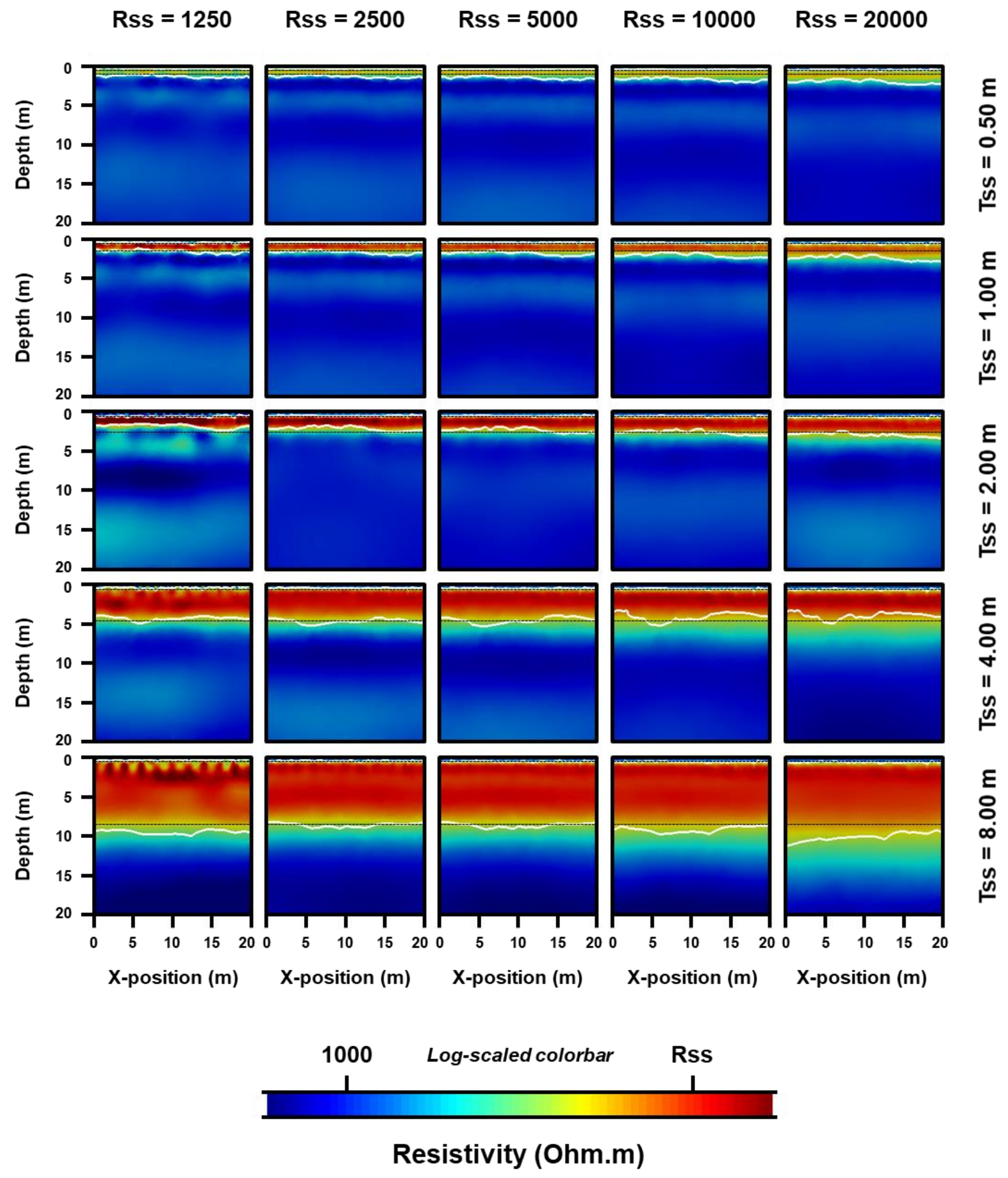

Figure S2: Results of inversion of the synthetic resistivity models (Rss and Tss values stand for the subsolum resistivity and thickness in the model, respectively; ERT-derived and true solum thickness and depth to bedrock interfaces are shown by white and black dashed lines, respectively) using the Wenner-Schlumberger array with an ES of $0.5 \mathrm{~m}$. 


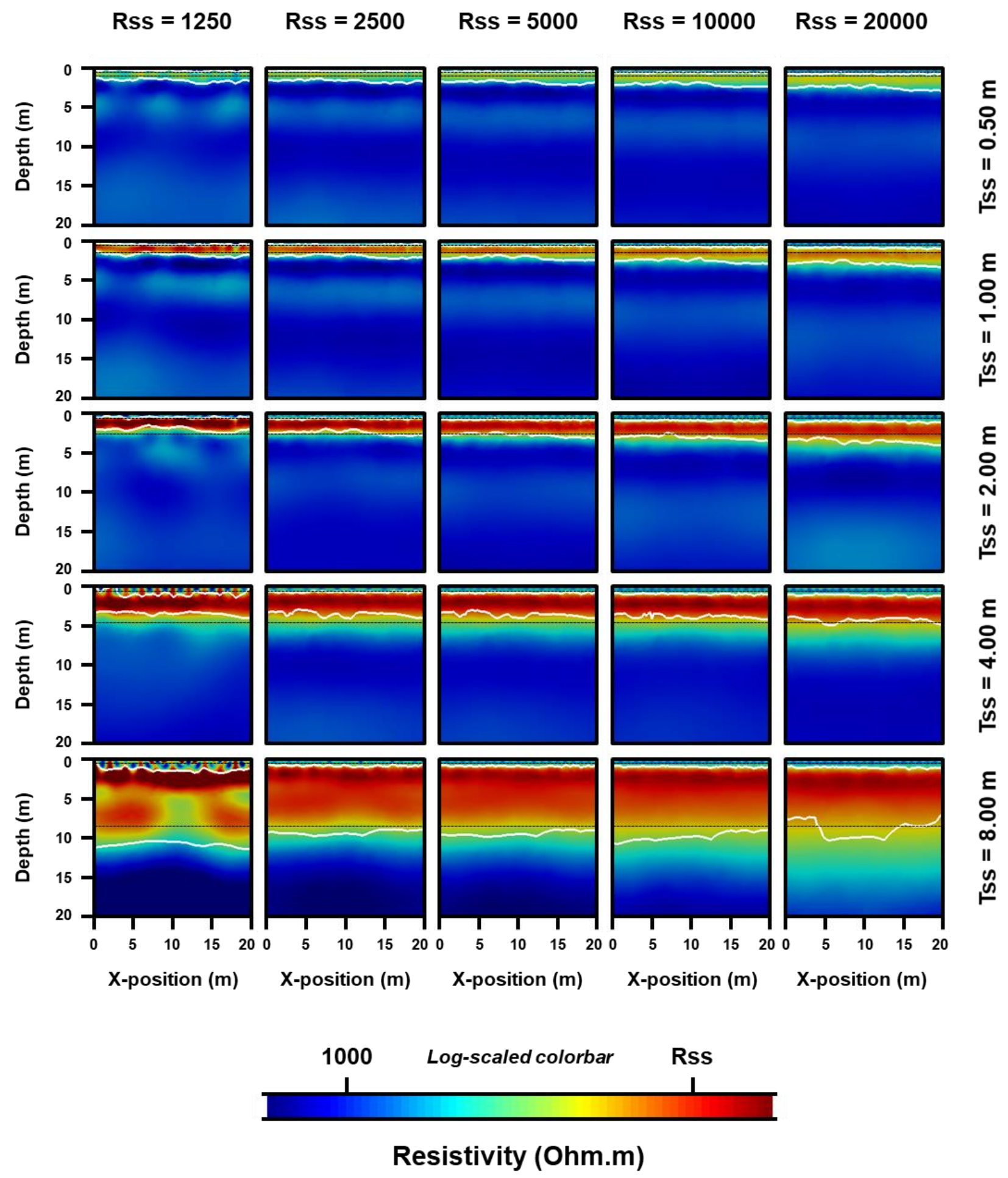

Figure S3: Results of inversion of the synthetic resistivity models (Rss and Tss values stand for the subsolum resistivity and thickness in the model, respectively; ERT-derived and true solum thickness and depth to bedrock interfaces are shown by white and black dashed lines, respectively) using the Wenner-Schlumberger array with an ES of $1 \mathrm{~m}$. 


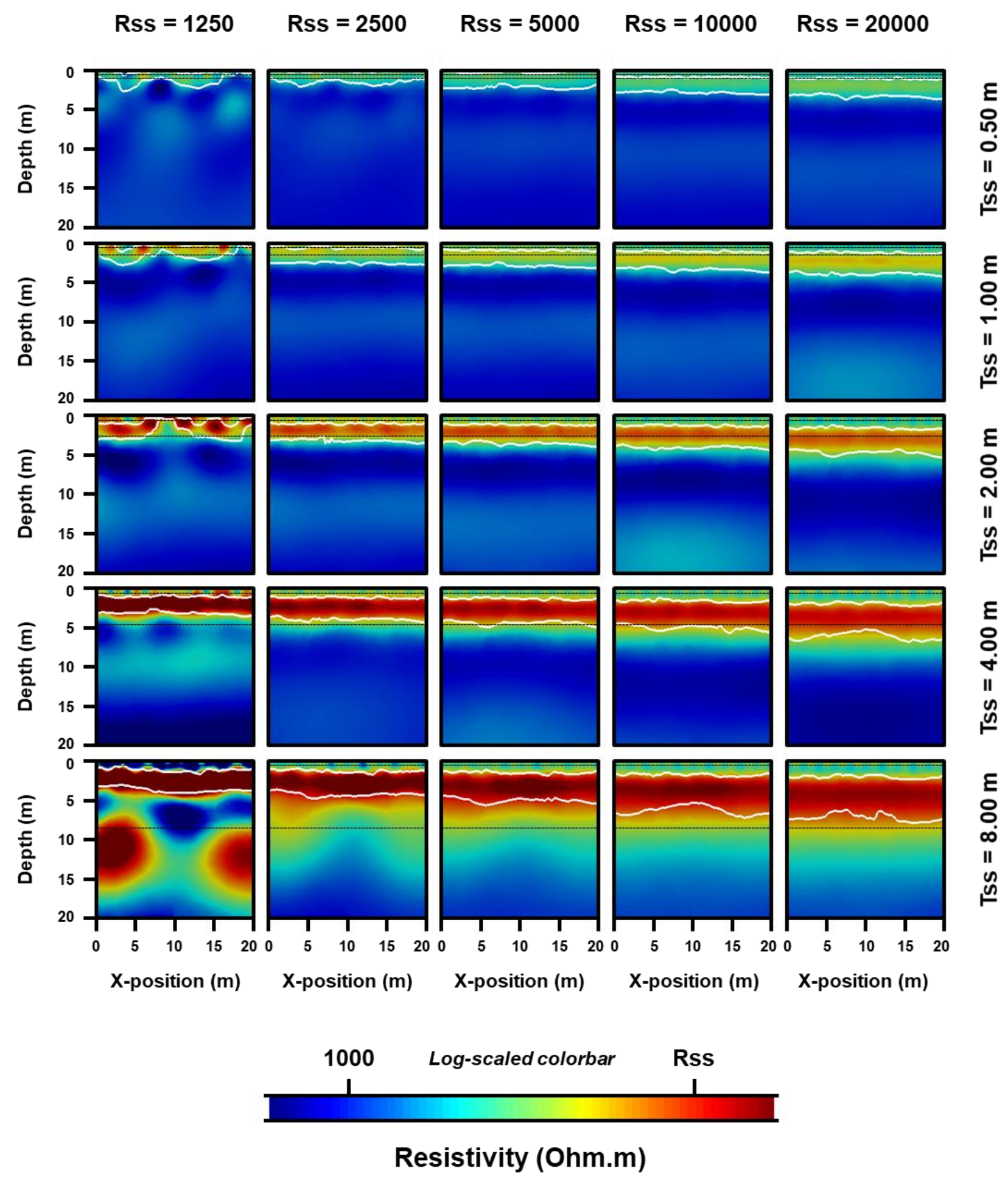

Figure S4: Results of inversion of the synthetic resistivity models (Rss and Tss values stand for the subsolum resistivity and thickness in the model, respectively; ERT-derived and true solum thickness and depth to bedrock interfaces are shown by white and black dashed lines, respectively) using the Wenner-Schlumberger array with an ES of $2 \mathrm{~m}$. 


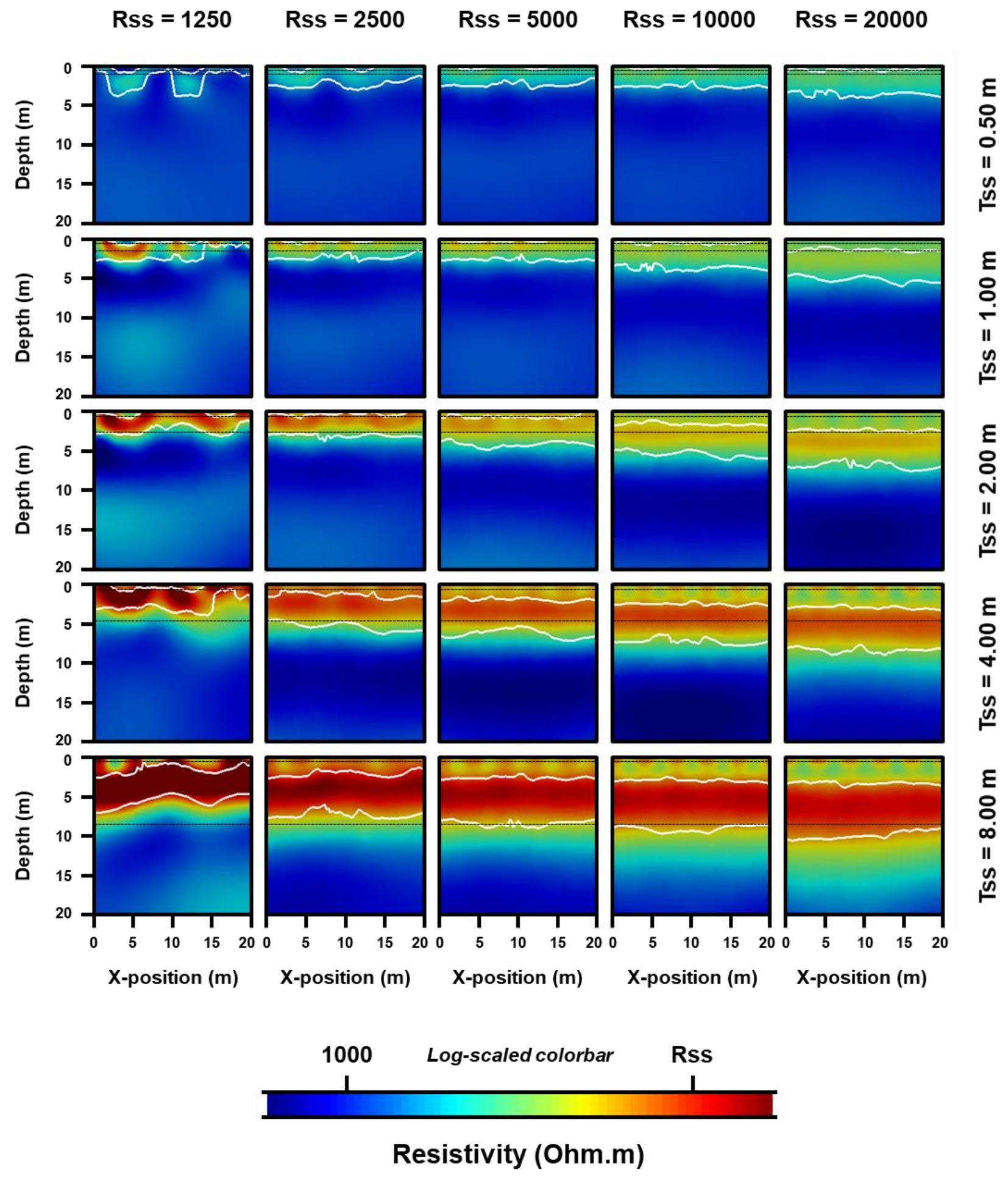

Figure S5: Results of inversion of the synthetic resistivity models (Rss and Tss values stand for the subsolum resistivity and thickness in the model, respectively; ERT-derived and true solum thickness and depth to bedrock interfaces are shown by white and black dashed lines, respectively) using the Wenner-Schlumberger array with an ES of $4 \mathrm{~m}$. 


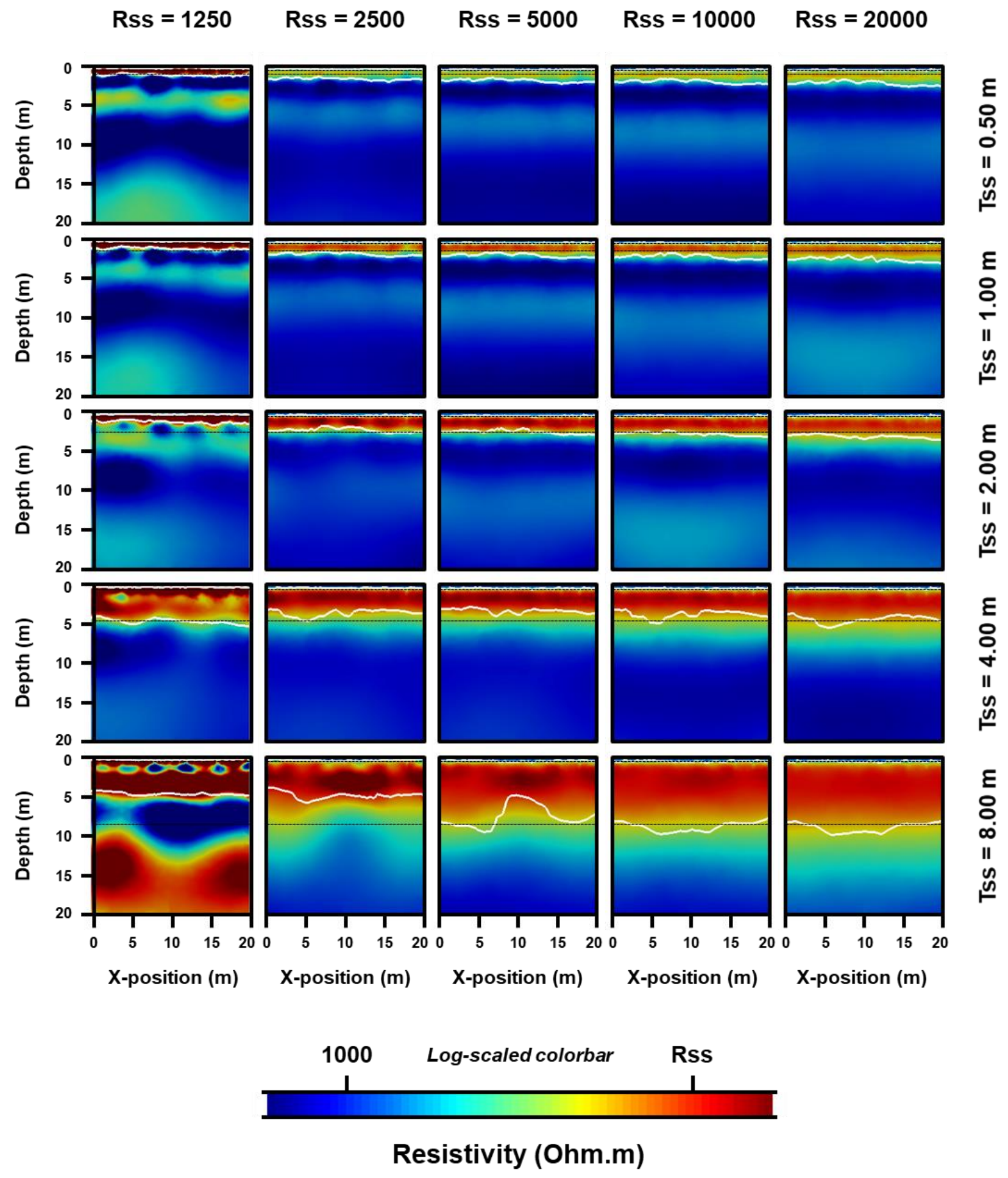

Figure S6: Results of inversion of the synthetic resistivity models (Rss and Tss values stand for the subsolum resistivity and thickness in the model, respectively; ERT-derived and true solum thickness and depth to bedrock true interfaces are shown by white and black dashed lines, respectively) using the Wenner-Schlumberger array with an ES of $2 \mathrm{~m}$ and upgraded with the four interpolated levels of surficial apparent resistivity. 


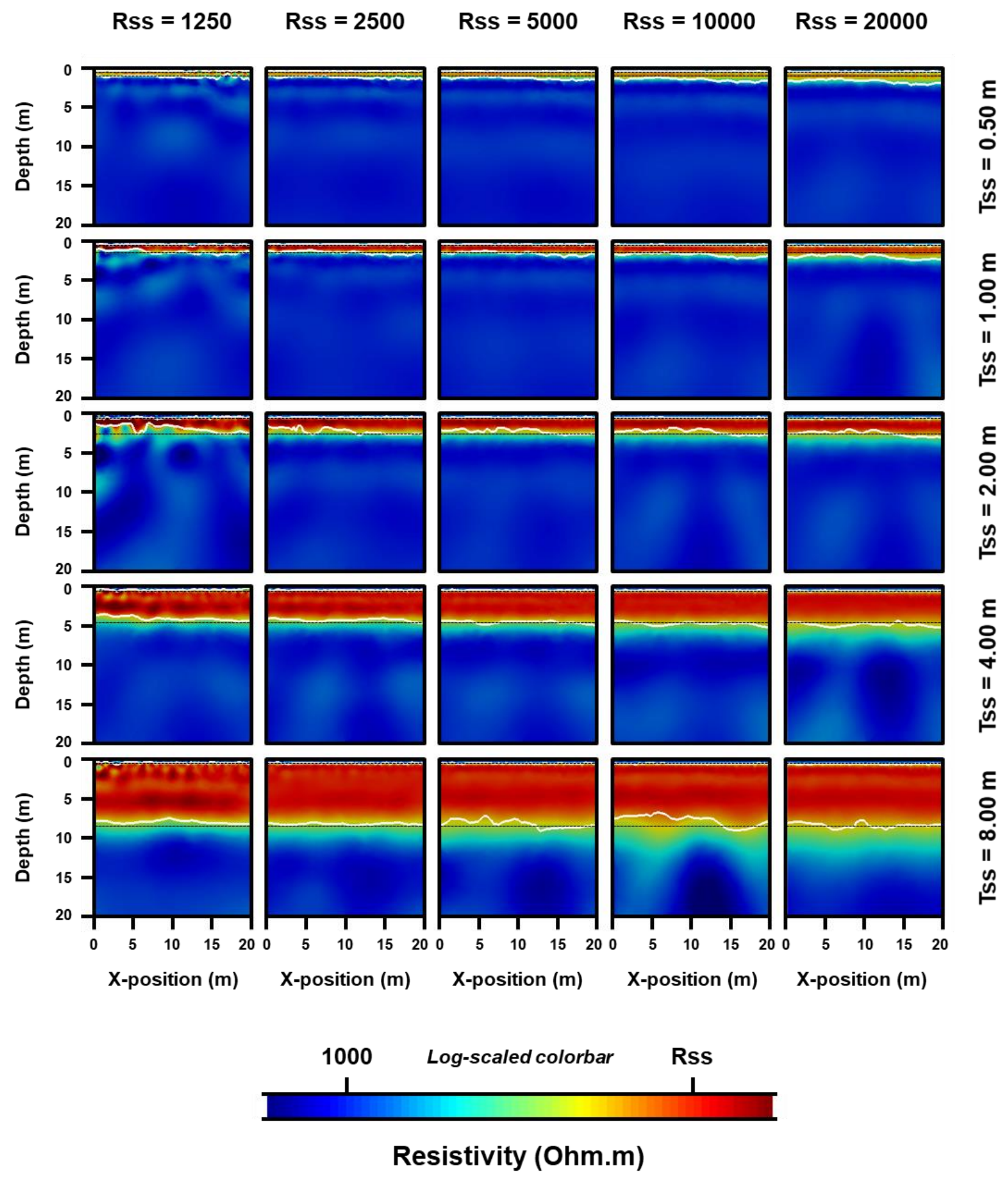

Figure S7: Results of inversion of the synthetic resistivity models (Rss and Tss values stand for the subsolum resistivity and thickness in the model, respectively; ERT-derived and true solum thickness and depth to bedrock interfaces are shown by white and black dashed lines, respectively) using the dipole-dipole array with an ES of $0.25 \mathrm{~m}$. 

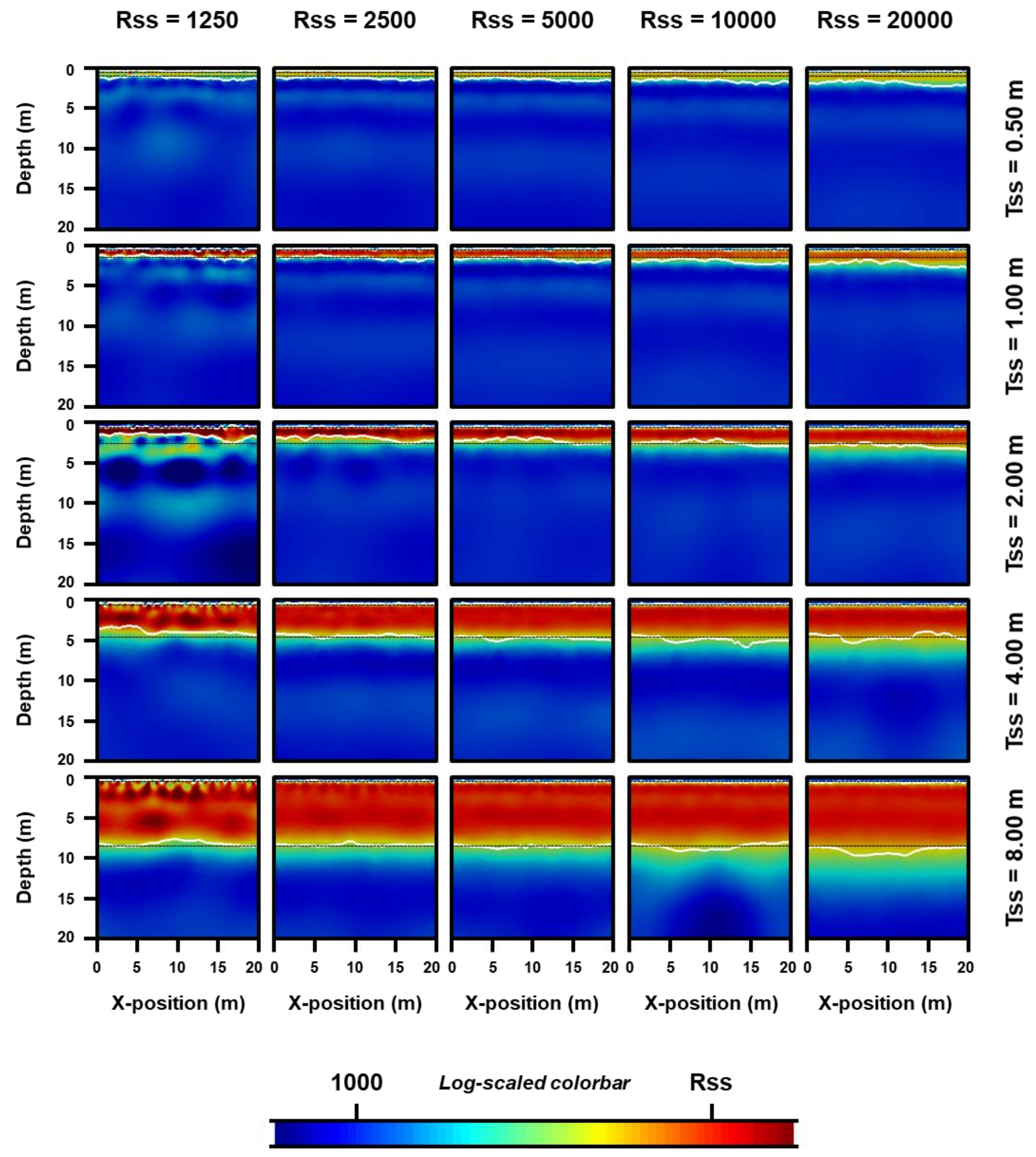

Resistivity (Ohm.m)

Figure S8: Results of inversion of the synthetic resistivity models (Rss and Tss values stand for the subsolum resistivity and thickness in the model, respectively; ERT-derived and true solum thickness and depth to bedrock interfaces are shown by white and black dashed lines, respectively) using the dipole-dipole array with an ES of $0.5 \mathrm{~m}$. 


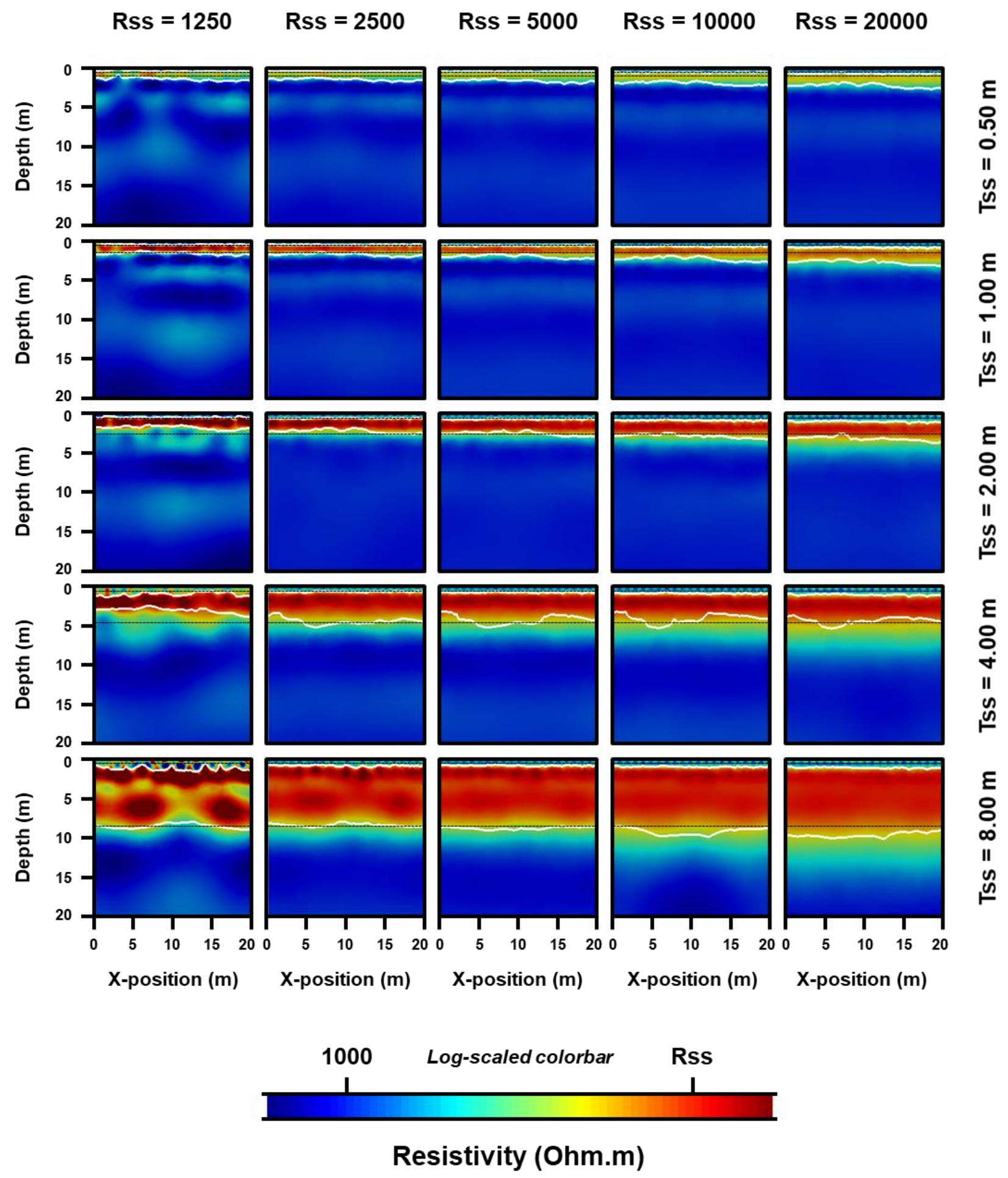

Figure S9: Results of inversion of the synthetic resistivity models (Rss and Tss values stand for the subsolum resistivity and thickness in the model, respectively; ERT-derived and true solum thickness and depth to bedrock interfaces are shown by white and black dashed lines, respectively) using the dipole-dipole array with an ES of $1 \mathrm{~m}$. 


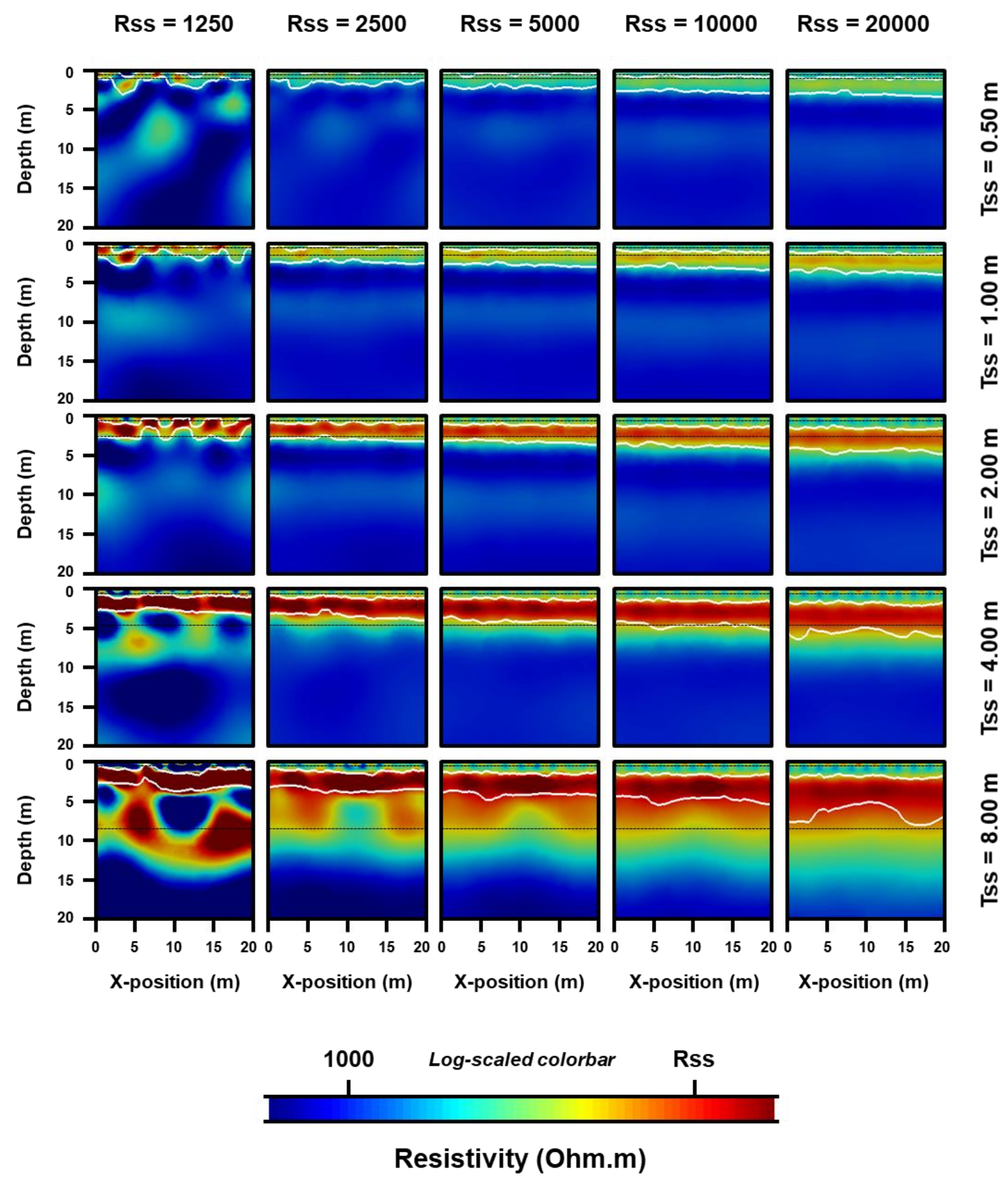

Figure S10: Results of inversion of the synthetic resistivity models (Rss and Tss values stand for the subsolum resistivity and thickness in the model, respectively; ERT-derived and true solum thickness and depth to bedrock interfaces are shown by white and black dashed lines, respectively) using the dipole-dipole array with an ES of $2 \mathrm{~m}$. 


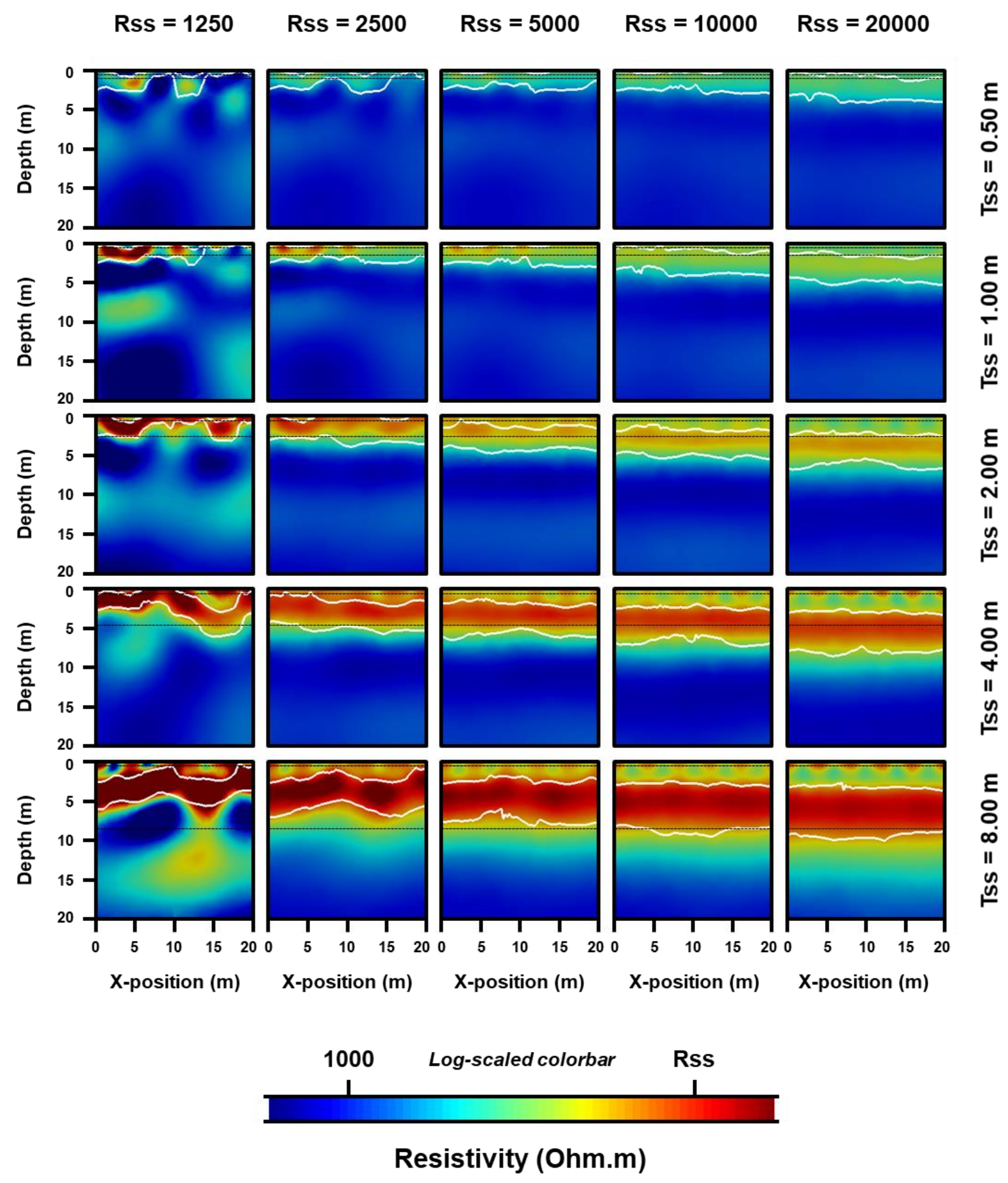

Figure S11: Results of inversion of the synthetic resistivity models (Rss and Tss values stand for the subsolum resistivity and thickness in the model, respectively; ERT-derived and true solum thickness and depth to bedrock interfaces are shown by white and black dashed lines, respectively) using the dipole-dipole array with an ES of $4 \mathrm{~m}$. 


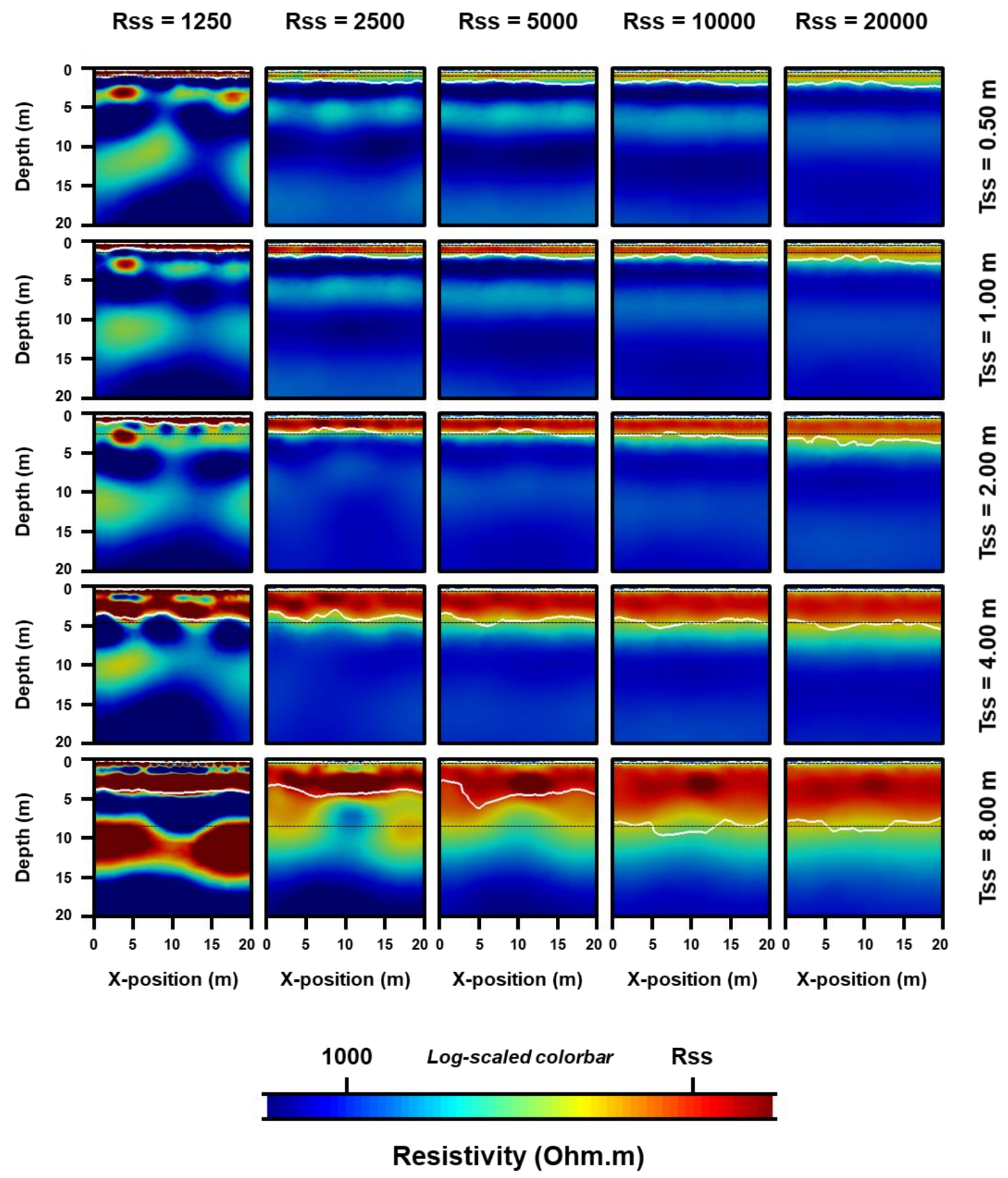

Figure S12: Results of inversion of the synthetic resistivity models (Rss and Tss values stand for the subsolum resistivity and thickness in the model, respectively; ERT-derived and true solum thickness and depth to bedrock interfaces are shown by white and black dashed lines, respectively) using the dipole-dipole array with an ES of $2 \mathrm{~m}$ and upgraded with the four interpolated levels of surficial apparent resistivity. 
Nash-Sutcliffe model efficiency coefficient
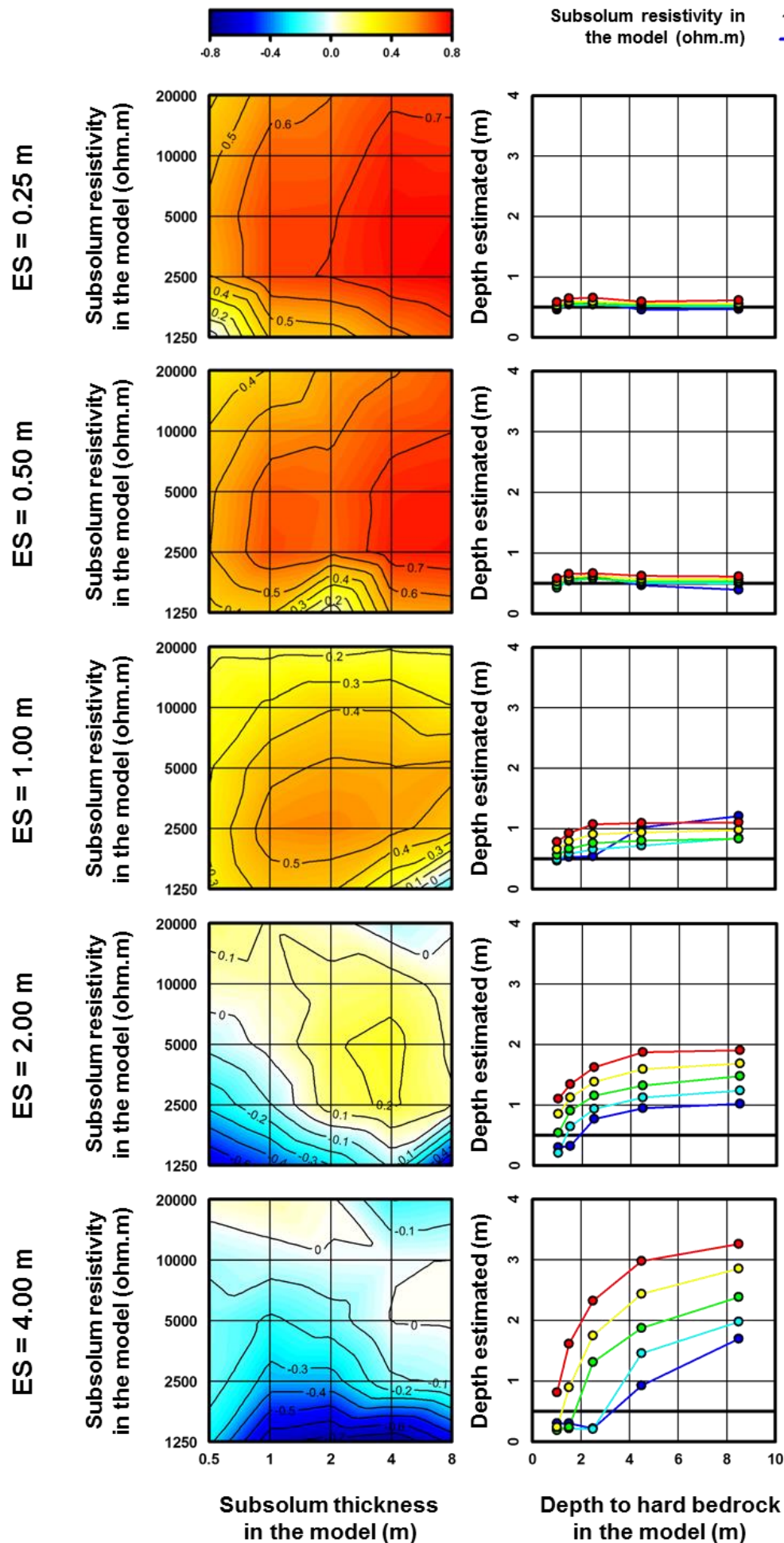

Solum thickness estimation

\section{Depth to hard bedrock estimation}
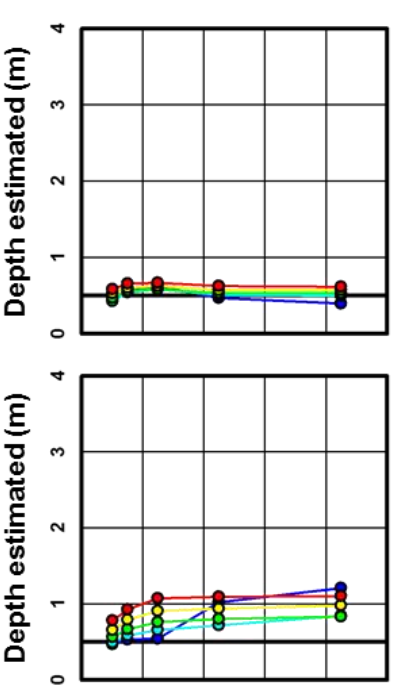

$\begin{array}{cccccc}\text { bsolum resistivity in } & 1250 & 2500 & 5000 & 10000 & 20000\end{array}$
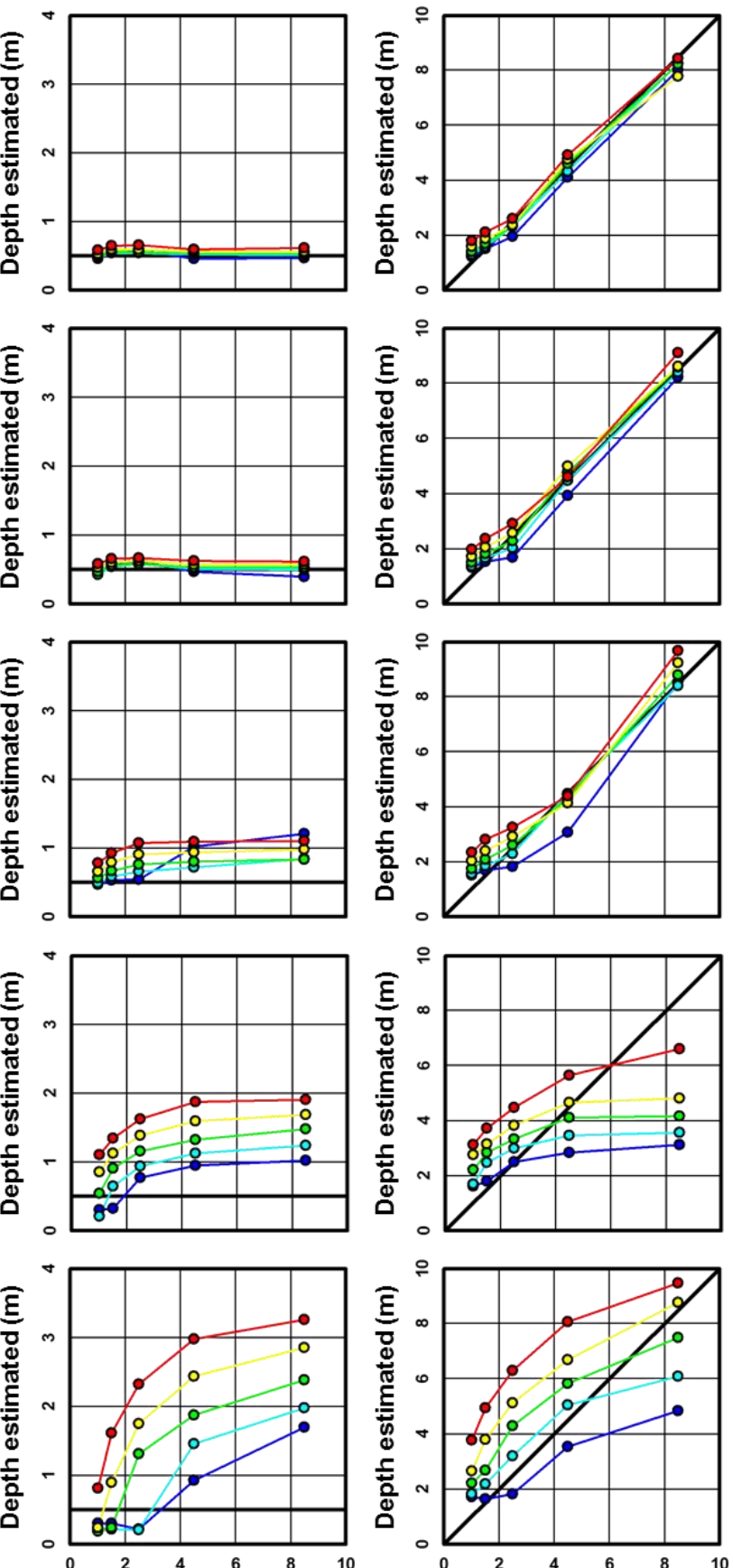

Depth to hard bedrock in the model $(\mathrm{m})$

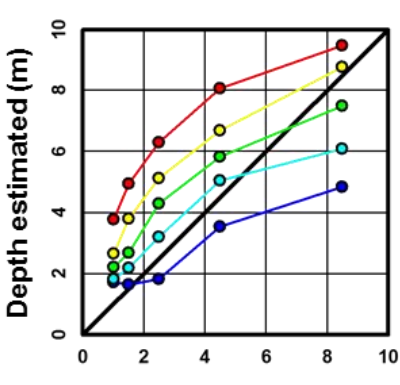

Depth to hard bedrock in the model $(\mathrm{m})$

Figure S13: Nash-Sutcliffe model efficiency coefficient and mean interface depths resulting from the inversion of the 25 synthetic apparent resistivity models using the dipole-dipole array with the five different ESs. In plots showing the estimated interface depths, thick black lines indicate the expected values. 


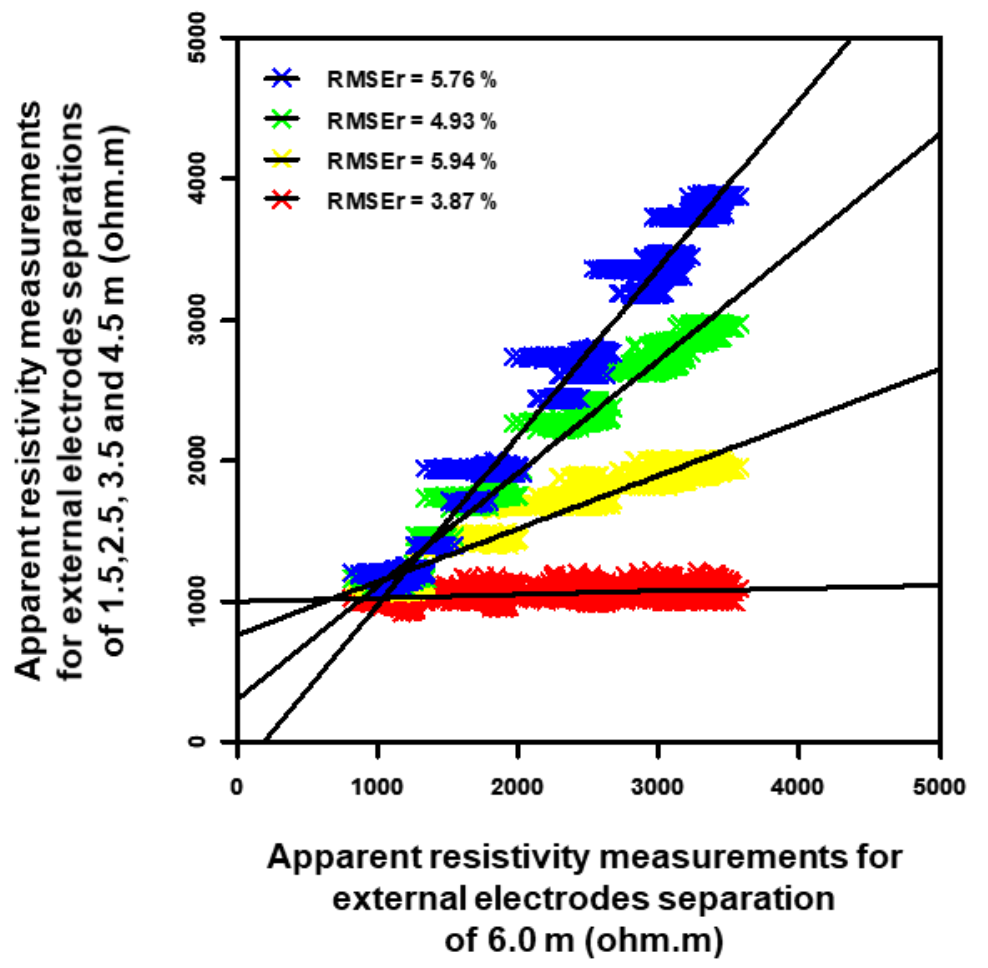

Figure S14: Scatter plot relating the apparent resistivity data corresponding to the first pseudo-depth acquisition level with an ES of $2 \mathrm{~m}$ (external electrodes spacing of $6 \mathrm{~m}$ ) versus the four surficial apparent resistivity levels using an ES of $0.5 \mathrm{~m}$ with external electrodes separations of 1.5 (red crosses), 2.5 (yellow crosses), 3.5 (green crosses) and $4.5 \mathrm{~m}$ (blue crosses) using the dipole-dipole array for the 25 synthetic resistivity models. Each point of the scatter plot represents the apparent resistivity of one synthetic model, for the same pseudo-x position, but at different pseudo-depths as defined by the external electrodes separation (see section 2.3 for more details). The linear regressions correspond to the thick black lines and their accuracy is indicated by the root mean square relative error (RMSEr). 


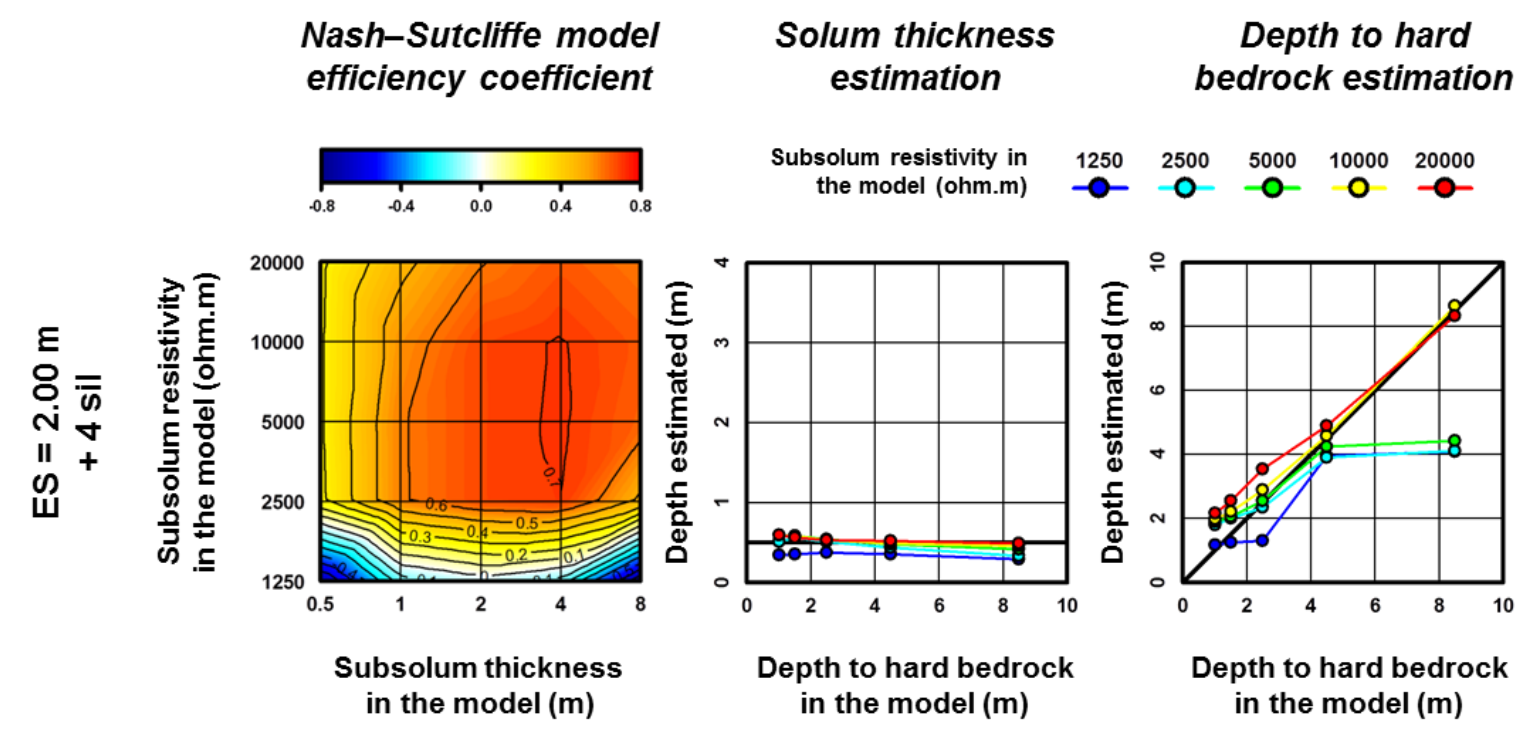

Figure S15: Nash-Sutcliffe model efficiency coefficient and mean interface depths resulting from the inversion of the 25 synthetic apparent resistivity models using the dipole-dipole array with an ES of $2 \mathrm{~m}$ and upgraded with the four interpolated levels of surficial apparent resistivity (sil stands for surficial interpolated levels). In plots showing the estimated interface depths, thick black lines indicate the expected values. 
Table S1: Nash-Sutcliffe model efficiency coefficient (NSE) and interface depths (avg \pm sd, average \pm standard deviation in $\mathrm{m}$; an italic value specifies that the interface delineation results from the merging of several second derivative zero contours) resulting from the inversion of the 25 synthetic apparent resistivity models (Tss, subsolum thickness in m; Rss, subsolum resistivity in ohm.m) using the dipole-dipole array with the 5 different ESs.

\section{$\mathrm{ES}=0.25 \mathrm{~m}$}

$\mathrm{ES}=0.50 \mathrm{~m}$

$\mathrm{ES}=1.00 \mathrm{~m}$

$\mathrm{ES}=2.00 \mathrm{~m}$

$\mathrm{ES}=4.00 \mathrm{~m}$

\begin{tabular}{|c|c|c|c|c|c|c|c|c|c|c|c|c|c|c|c|c|c|c|c|c|c|c|c|c|c|c|c|c|c|c|c|c|c|c|c|c|}
\hline \multirow[t]{2}{*}{ Tss } & \multirow[t]{2}{*}{ Rss } & \multirow[t]{2}{*}{ NSE } & \multicolumn{3}{|c|}{$\begin{array}{l}\text { Solum } \\
\text { depth }\end{array}$} & \multicolumn{3}{|c|}{$\begin{array}{l}\text { Depth to } \\
\text { bedrock }\end{array}$} & \multirow[t]{2}{*}{ NSE } & \multicolumn{3}{|c|}{$\begin{array}{c}\text { Solum } \\
\text { depth }\end{array}$} & \multicolumn{3}{|c|}{$\begin{array}{l}\text { Depth to } \\
\text { bedrock }\end{array}$} & \multirow[t]{2}{*}{ NSE } & \multicolumn{3}{|c|}{$\begin{array}{l}\text { Solum } \\
\text { depth }\end{array}$} & \multicolumn{3}{|c|}{$\begin{array}{l}\text { Depth to } \\
\text { bedrock }\end{array}$} & \multirow[t]{2}{*}{ NSE } & \multicolumn{3}{|c|}{$\begin{array}{l}\text { Solum } \\
\text { depth }\end{array}$} & \multicolumn{3}{|c|}{$\begin{array}{l}\text { Depth to } \\
\text { bedrock }\end{array}$} & \multirow[t]{2}{*}{ NSE } & \multicolumn{3}{|c|}{$\begin{array}{l}\text { Solum } \\
\text { depth }\end{array}$} & \multicolumn{3}{|c|}{$\begin{array}{l}\text { Depth to } \\
\text { bedrock }\end{array}$} \\
\hline & & & avg & & & avg & + & & & avg & \pm & & avg & & & & avg & & & avg & & & & avg & + & & avg & $\pm s$ & & & avg & \pm & & avg & \pm & \\
\hline 0.5 & 1250 & -0.07 & 0.46 & \pm & 0.08 & 1.22 & \pm & 0.15 & 0.36 & 0.44 & \pm & 0.07 & 1.35 & & 0.16 & 0.28 & 0.47 & \pm & 0.08 & 1.51 & + & 0.19 & -0.61 & 0.31 & \pm & 0.32 & 1.62 & \pm 0 & 0.61 & -0.27 & 0.30 & \pm & 0.34 & 1.73 & \pm & 1.09 \\
\hline 0.5 & 2500 & 0.50 & 0.48 & \pm & 0.05 & 1.31 & \pm & 0.13 & 0.50 & 0.44 & \pm & 0.03 & 1.39 & & 0.14 & 0.32 & 0.49 & \pm & 0.04 & 1.58 & & 0.15 & -0.27 & 0.21 & \pm & 0.15 & 1.71 & \pm 0 & 0.31 & -0.14 & 0.23 & \pm & 0.27 & 1.83 & \pm & 0.82 \\
\hline 0.5 & 5000 & 0.53 & 0.51 & \pm & 0.03 & 1.42 & \pm & 0.15 & 0.48 & 0.47 & \pm & 0.04 & 1.52 & & 0.15 & 0.29 & 0.56 & \pm & 0.04 & 1.75 & 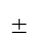 & 0.17 & -0.07 & 0.54 & \pm & 0.17 & 2.21 & \pm 0 & 0.18 & -0.13 & 0.19 & \pm & 0.21 & 2.21 & \pm & 0.37 \\
\hline 0.5 & 10000 & 0.47 & 0.54 & \pm & 0.03 & 1.59 & \pm & 0.18 & 0.41 & 0.53 & \pm & 0.04 & 1.72 & \pm & 0.19 & 0.24 & 0.66 & \pm & 0.05 & 2.03 & \pm & 0.21 & 0.06 & 0.86 & \pm & 0.11 & 2.77 & \pm 0 & 0.15 & -0.09 & 0.24 & \pm & 0.19 & 2.65 & \pm & 0.31 \\
\hline 0.5 & 20000 & 0.38 & 0.59 & \pm & 0.03 & 1.81 & \pm & 0.23 & 0.30 & 0.59 & \pm & 0.04 & 1.99 & \pm & 0.24 & 0.18 & 0.79 & \pm & 0.06 & 2.36 & \pm & 0.26 & 0.13 & 1.11 & \pm & 0.09 & 3.14 & \pm 0 & 0.21 & 0.05 & 0.82 & \pm & 0.34 & 3.77 & \pm & 0.38 \\
\hline 1 & 1250 & 0.42 & 0.56 & \pm & 0.06 & 1.51 & + & 0.23 & 0.43 & 0.59 & \pm & 0.08 & 1.53 & & 0.18 & 0.44 & 0.53 & \pm & 0.09 & 1.69 & & 0.15 & -0.46 & 0.33 & \pm & 0.37 & 1.80 & \pm 0 & 0.63 & -0.67 & 0.30 & \pm & 0.27 & 1.65 & \pm & 0.80 \\
\hline 1 & 2500 & 0.68 & 0.55 & \pm & 0.03 & 1.59 & \pm & 0.18 & 0.67 & 0.55 & \pm & 0.04 & 1.67 & & 0.18 & 0.56 & 0.58 & \pm & 0.04 & 1.85 & + & 0.18 & -0.10 & 0.65 & \pm & 0.20 & 2.47 & \pm 0 & 0.17 & -0.34 & 0.22 & \pm & 0.18 & 2.20 & \pm & \\
\hline 1 & 5000 & 0.68 & 0.56 & \pm & 0.03 & 1.70 & \pm & 0.20 & 0.65 & 0.57 & \pm & 0.04 & 1.83 & \pm & 0.21 & 0.47 & 0.67 & \pm & 0.05 & 2.09 & \pm & 0.21 & 0.03 & 0.91 & \pm & 0.10 & 2.83 & \pm 0 & 0.16 & -0.22 & 0.24 & \pm & 0.19 & 2.68 & \pm & 0.35 \\
\hline 1 & 10000 & 0.64 & 0.60 & \pm & 0.02 & 1.88 & \pm & 0.23 & 0.56 & 0.61 & \pm & 0.04 & 2.07 & \pm & 0.24 & 0.32 & 0.79 & \pm & 0.06 & 2.40 & \pm & 0.27 & 0.08 & 1.12 & \pm & 0.09 & 3.17 & \pm 0 & 0.21 & -0.05 & 0.90 & \pm & 0.32 & 3.81 & \pm & 0.40 \\
\hline 1 & 20000 & 0.56 & 0.65 & \pm & 0.03 & 2.11 & \pm & 0.26 & 0.41 & 0.66 & \pm & 0.05 & 2.38 & \pm & 0.31 & 0.17 & 0.93 & \pm & 0.07 & 2.81 & \pm & 0.26 & 0.10 & 1.35 & \pm & 0.12 & 3.73 & \pm 0 & 0.23 & 0.08 & 1.61 & \pm & 0.27 & 4.95 & \pm & 0.28 \\
\hline 2 & 1250 & 0.44 & 0.59 & \pm & 0.09 & 1.97 & \pm & 0.45 & 0.01 & 0.62 & \pm & 0.10 & 1.71 & & 0.34 & 0.42 & 0.54 & \pm & 0.09 & 1.84 & \pm & 0.23 & -0.31 & 0.77 & \pm & 0.41 & 2.50 & \pm 0 & 0.61 & -0.74 & 0.22 & \pm & 0.30 & 1.84 & \pm & 0.92 \\
\hline 2 & 2500 & 0.71 & 0.55 & \pm & 0.04 & 2.32 & \pm & 0.37 & 0.63 & 0.59 & \pm & 0.06 & 2.03 & \pm & 0.31 & 0.58 & 0.66 & \pm & 0.05 & 2.29 & \pm & 0.25 & 0.16 & 0.94 & \pm & 0.15 & 3.01 & \pm 0 & 0.19 & -0.34 & 0.21 & \pm & 0.27 & 3.22 & \pm & 0.39 \\
\hline 2 & 5000 & 0.69 & 0.56 & \pm & 0.04 & 2.34 & \pm & 0.28 & 0.63 & 0.59 & \pm & 0.05 & 2.31 & \pm & 0.28 & 0.52 & 0.76 & \pm & 0.06 & 2.61 & \pm & 0.25 & 0.19 & 1.16 & \pm & 0.09 & 3.34 & $\pm c$ & 0.19 & -0.15 & 1.32 & \pm & 0.30 & 4.30 & \pm & 0.25 \\
\hline 2 & 10000 & 0.64 & 0.59 & \pm & 0.04 & 2.38 & \pm & 0.30 & 0.58 & 0.63 & \pm & 0.05 & 2.59 & - & 0.27 & 0.39 & 0.91 & \pm & 0.07 & 2.91 & \pm & 0.23 & 0.13 & 1.39 & \pm & 0.11 & 3.83 & \pm 0 & 0.21 & -0.01 & 1.75 & \pm & 0.26 & 5.13 & \pm & 0.28 \\
\hline 2 & 20000 & 0.60 & 0.66 & \pm & 0.04 & 2.60 & \pm & 0.30 & 0.53 & 0.67 & \pm & 0.06 & 2.93 & 1 & 0.23 & 0.16 & 1.08 & \pm & 0.08 & 3.25 & \pm & 0.27 & 0.04 & 1.63 & \pm & 0.14 & 4.47 & \pm 0 & 0.23 & 0.02 & 2.32 & \pm & 0.18 & 6.31 & \pm & 0.47 \\
\hline 4 & 1250 & 0.57 & 0.47 & \pm & 0.09 & 4.11 & \pm & 0.25 & 0.51 & 0.47 & \pm & 0.15 & 3.92 & & 0.33 & 0.19 & 1.02 & \pm & 0.24 & 3.09 & \pm & 0.34 & -0.08 & 0.95 & \pm & 0.14 & 2.85 & \pm 0 & 0.23 & -0.93 & 0.93 & \pm & 0.95 & 3.55 & \pm & 1.52 \\
\hline 4 & 2500 & 0.77 & 0.53 & \pm & 0.04 & 4.32 & \pm & 0.16 & 0.75 & 0.51 & \pm & 0.06 & 4.49 & & 0.16 & 0.53 & 0.72 & \pm & 0.07 & 4.49 & - & 0.43 & 0.22 & 1.13 & \pm & 0.14 & 3.47 & \pm 0 & 0.37 & -0.11 & 1.46 & \pm & 0.50 & 5.06 & \pm & 0.41 \\
\hline 4 & 5000 & 0.77 & 0.54 & \pm & 0.04 & 4.61 & \pm & 0.16 & 0.74 & 0.54 & \pm & 0.05 & 4.78 & & 0.17 & 0.51 & 0.81 & \pm & 0.07 & 4.31 & $\stackrel{1}{2}$ & 0.59 & 0.24 & 1.33 & \pm & 0.14 & 4.12 & \pm 0 & 0.20 & 0.01 & 1.88 & \pm & 0.29 & 5.84 & \pm & 0.39 \\
\hline 4 & 10000 & 0.75 & 0.57 & \pm & 0.04 & 4.78 & \pm & 0.21 & 0.67 & 0.57 & \pm & 0.05 & 5.01 & - & 0.35 & 0.39 & 0.94 & \pm & 0.07 & 4.14 & 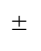 & 0.60 & 0.16 & 1.59 & \pm & 0.15 & 4.67 & \pm 0 & 0.28 & -0.01 & 2.43 & \pm & 0.18 & 6.70 & \pm & 0.48 \\
\hline 4 & 20000 & 0.68 & 0.60 & \pm & 0.04 & 4.92 & \pm & 0.21 & 0.59 & 0.63 & \pm & 0.05 & 4.62 & 1 & 0.38 & 0.19 & 1.10 & \pm & 0.08 & 4.40 & 1 & 0.44 & -0.08 & 1.88 & \pm & 0.18 & 5.64 & \pm 0 & 0.41 & -0.21 & 2.97 & \pm & 0.17 & 8.06 & \pm & 0.25 \\
\hline 8 & 1250 & 0.65 & 0.47 & \pm & 0.08 & 8.01 & $I$ & 0.19 & 0.54 & 0.40 & \pm & 0.14 & 8.23 & & 0.28 & -0.24 & 1.21 & \pm & 0.26 & 8.54 & 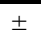 & 0.30 & -0.65 & 1.03 & \pm & 0.32 & 3.13 & \pm 0 & 0.42 & -0.58 & 1.70 & \pm & 0.67 & 4.85 & \pm & 0.68 \\
\hline 8 & 2500 & 0.80 & 0.52 & \pm & 0.04 & 8.25 & \pm & 0.10 & 0.77 & 0.50 & \pm & 0.05 & 8.39 & & 0.12 & 0.44 & 0.85 & \pm & 0.10 & 8.40 & - & 0.19 & 0.11 & 1.25 & \pm & 0.19 & 3.57 & \pm 0 & 0.29 & -0.08 & 1.98 & \pm & 0.55 & 6.08 & \pm & 0.67 \\
\hline 8 & 5000 & 0.78 & 0.54 & \pm & 0.04 & 8.23 & \pm & 0.48 & 0.75 & 0.54 & \pm & 0.05 & 8.58 & - & 0.14 & 0.52 & 0.84 & \pm & 0.07 & 8.79 & - & 0.16 & 0.08 & 1.48 & \pm & 0.13 & 4.16 & \pm 0 & 0.28 & 0.01 & 2.38 & \pm & 0.33 & 7.50 & \pm & 0.56 \\
\hline 8 & 10000 & 0.73 & 0.57 & \pm & 0.04 & 7.77 & - & 0.68 & 0.70 & 0.58 & \pm & 0.05 & 8.61 & - & 0.36 & 0.30 & 0.98 & \pm & 0.06 & 9.22 & - & 0.42 & 0.05 & 1.69 & \pm & 0.13 & 4.82 & \pm 0 & 0.34 & 0.00 & 2.85 & \pm & 0.17 & 8.78 & \pm & 0.44 \\
\hline 8 & 20000 & 0.69 & 0.61 & \pm & 0.04 & 8.42 & \pm & 0.30 & 0.66 & 0.62 & \pm & 0.05 & 9.12 & 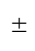 & 0.40 & 0.18 & 1.10 & \pm & 0.08 & 9.66 & \pm & 0.31 & -0.01 & 1.90 & \pm & 0.18 & 6.61 & \pm 1 & 1.04 & -0.17 & 3.25 & \pm & 0.20 & 9.48 & \pm & 0.35 \\
\hline
\end{tabular}


Table S2: Nash-Sutcliffe model efficiency coefficient (NSE) and interface depths (avg \pm sd, average \pm standard deviation in $\mathrm{m}$; an italic value specifies that the interface delineation has gone through the merging of several second derivative zero contours) resulting from the inversion of the 25 synthetic apparent resistivity models (Tss, subsolum thickness in m; Rss, subsolum resistivity in ohm.m) using the dipole-dipole array with the ES of $2 \mathrm{~m}$ upgraded with the four interpolated levels of surficial apparent resistivity (sil stands for surficial interpolated levels).

$$
\mathrm{ES}=2.00 \mathrm{~m}+4 \text { sil }
$$

\begin{tabular}{|c|c|c|c|c|c|c|c|c|}
\hline \multirow[t]{2}{*}{ Tss } & \multirow[t]{2}{*}{ Rss } & \multirow[t]{2}{*}{ NSE } & \multicolumn{3}{|c|}{$\begin{array}{l}\text { Solum } \\
\text { depth }\end{array}$} & \multicolumn{3}{|c|}{$\begin{array}{l}\text { Depth to } \\
\text { bedrock }\end{array}$} \\
\hline & & & avg & \pm & & avg & $\pm s$ & \\
\hline 0.5 & 1250 & -0.74 & 0.35 & \pm & 0.05 & 1.18 & \pm 0 & 0.10 \\
\hline 0.5 & 2500 & 0.28 & 0.51 & \pm & 0.09 & 1.82 & \pm 0 & 0.12 \\
\hline 0.5 & 5000 & 0.29 & 0.60 & + & 0.05 & 1.91 & \pm 0 & 0.17 \\
\hline 0.5 & 10000 & 0.29 & 0.59 & \pm & 0.04 & 1.98 & \pm 0 & 0.20 \\
\hline 0.5 & 20000 & 0.28 & 0.60 & \pm & 0.05 & 2.16 & \pm & 0.24 \\
\hline 1 & 1250 & -0.15 & 0.36 & \pm & 0.04 & 1.26 & \pm & 0.14 \\
\hline 1 & 2500 & 0.59 & 0.57 & \pm & 0.07 & 2.01 & \pm & 0.16 \\
\hline 1 & 5000 & 0.59 & 0.59 & \pm & 0.05 & 2.07 & \pm & 0.20 \\
\hline 1 & 10000 & 0.56 & 0.58 & \pm & 0.05 & 2.21 & \pm & 0.25 \\
\hline 1 & 20000 & 0.47 & 0.57 & \pm & 0.05 & 2.57 & \pm & 0.29 \\
\hline 2 & 1250 & -0.05 & 0.38 & 1 & 0.04 & 1.31 & \pm & 0.17 \\
\hline 2 & 2500 & 0.68 & 0.52 & \pm & 0.07 & 2.35 & \pm & 0.25 \\
\hline 2 & 5000 & 0.68 & 0.54 & \pm & 0.06 & 2.55 & \pm & 0.26 \\
\hline 2 & 10000 & 0.68 & 0.53 & \pm & 0.05 & 2.89 & \pm & 0.21 \\
\hline 2 & 20000 & 0.59 & 0.54 & \pm & 0.05 & 3.53 & \pm & 0.31 \\
\hline 4 & 1250 & -0.14 & 0.36 & \pm & 0.05 & 3.96 & \pm & 0.29 \\
\hline 4 & 2500 & 0.70 & 0.44 & \pm & 0.05 & 3.90 & \pm & 0.36 \\
\hline 4 & 5000 & 0.71 & 0.48 & \pm & 0.05 & 4.25 & \pm & 0.38 \\
\hline 4 & 10000 & 0.70 & 0.49 & \pm & 0.06 & 4.57 & \pm & 0.37 \\
\hline 4 & 20000 & 0.65 & 0.52 & \pm & 0.05 & 4.90 & \pm & 0.35 \\
\hline 8 & 1250 & -1.56 & 0.29 & - & 0.11 & 4.09 & \pm & 0.15 \\
\hline 8 & 2500 & 0.48 & 0.34 & \pm & 0.11 & 4.13 & \pm & 0.41 \\
\hline 8 & 5000 & 0.63 & 0.43 & \pm & 0.08 & 4.42 & \pm & 0.84 \\
\hline 8 & 10000 & 0.65 & 0.47 & \pm & 0.06 & 8.64 & \pm & 0.63 \\
\hline 8 & 20000 & 0.60 & 0.50 & \pm & 0.06 & 8.33 & \pm & 0.55 \\
\hline
\end{tabular}

